\title{
Pre-purification of Plantago lanceolata extracts with biologically active compounds using yeast cells
}

\author{
Vladimír Štefuca ${ }^{a}$, Lukáš Rada ${ }^{a}$, \\ Alžbeta Chochulováb ${ }^{\text {, Michal Rosenberg }}{ }^{a}$ \\ ${ }^{a}$ Institute of Biotechnology, Faculty of Chemical and Food Technology, Slovak University of Technology, \\ Radlinského 9, SK-812 37 Bratislava, Slovakia \\ ${ }^{b}$ Axxence Slovakia, s.r.o., Mickiewiczova 9, SK-811 07 Bratislava, Slovakia \\ vladimir.stefuca@stuba.sk
}

\begin{abstract}
Leaves of the plant Plantago lanceolata contain many economically interesting bioactive compounds, among them aucubin and catalpol are the most attractive. However, soluble saccharides passing to water extracts during isolation complicate chromatographic purification of these compounds. Their degradation by microbial cells transforming, for example, glucose, fructose, or sucrose to ethanol could bring important production costs savings and improved final product quality. It has been shown that the best saccharide degradation in extracts is achieved with the Saccharomyces cerevisiae cells. The cells were very active also in their immobilized form and they were able to completely remove glucose from the extract within four hours in a packed bed reactor combined with a stirring system with infinite medium recirculation.

A simple mathematical model involving reaction kinetics and mass transfer limitations in the cell particles was proposed for the evaluation of cell effectiveness in their immobilized form in term of effectiveness factor. Values of the effectiveness factor calculated from the model were far below 1, indicating strong mass transfer limitations of the reaction. The model is suitable for optimization of preparation of immobilized cell particles, mainly from the point of view of cell charge in particles.
\end{abstract}

Keywords: Plantago lanceolata, bioactive compounds, pre-purification method, immobilized cells, saccharide removal

\section{Introduction}

Positive medical effects of Plantago plants have been known for a very long time. Extensive traditional use and modern medicinal application of Plantago species is the result of the remarkable variety of medical properties: astringent, styptic, antimicrobial, expectorant, diuretic, and demulcent. In addition, some studies confirm that certain Plantago species show considerable bioactivity, such as cytotoxic effects on cancer cell lines, as well as antiinflammatory, immunoregulatory, antioxidant, and antispasmodic effects. Due to these properties, Plantago lanceolata, which is one of the typical species, is a very attractive plant that is used both in traditional healing and in current conventional therapy. Therefore, from a practical point of view, it is worthwhile to consider Plantago lanceolata for the isolation of economically interesting compounds; e.g.: phenylethanoids like acteoside, cistanoside F, lavandulifolioside, plantamajoside, flavonoids like apigenin, quercetin, plantaginin, and iridoid glucosides such as aucubin and catalpol.

The above mentioned iridoids are associated with a wide range of health benefits. They provide prevention against a broad range of physical, chemical, or biological stressors. They have a normalizing effect on the human body targeting imbalance without affecting proper functioning of the systems. Phar- macological studies showed that iridoid glucosides, particularly aucubin, have diverse biological activities. Aucubin conversion to its aglucone form seems to be a prerequisite step for the inhibition of ethoxy coumarin O-deethylase (Chang, 1997).

Aucubin also inhibits the release of leukotriene $\mathrm{C} 4$ and thromboxane B2 from calcium ionophorestimulated samples (Bermejo Benito et al., 2000) and the activation of nuclear factor-kappa $B$ in the mast cells, which might explain its beneficial effect in the treatment of chronic allergic inflammatory diseases (Kupeli et al., 2007).

Recently, aucubin was found to be a novel topoisomerase I inhibitor which increased the interest in its possible use in cancer chemoprevention and antitumoral therapy (Galvez et al., 2005).

Specially, $P$. lanceolata is characteristic for its content of iridoid glucosides: aucubin: $0.68 \pm 0.23 \%$, catalpol: $0.89 \pm 0.22 \%$ (Gonda et al., 2010).

Such low product concentration in the raw material leads to the development of economically favorable methods and isolation techniques putting emphasis on the plant biomass treatment, shortcutting the way toward much more concentrated material to reduce volumes entering the following isolation stages. Especially in case of aucubin preparation, reducing the extract volume results in high concentrations of soluble saccharides, which complicates the chromatographic separation steps. 
Therefore, the aim of this paper was to develop and characterize a method of mono- and disaccharides elimination in the extract by means of metabolic activities of free and immobilized yeasts as well as to propose a simple mathematical model enabling the evaluation of the effectiveness of immobilized cell application.

\section{Theoretical}

\section{Mathematic model}

Mathematic model described in this section was used for the evaluation of kinetic measurements performed in the immobilized cell system with infinite recirculation as shown in Fig. 1. Considering a simple reaction, $\mathrm{S} \rightarrow \mathrm{P}$, the reaction rate in porous spherical catalytic particles in steady state can be generally calculated from the equation

$$
D_{e S}\left(\frac{\partial^{2} c_{S}}{\partial r^{2}}+\frac{2}{r} \frac{\partial c_{S}}{\partial r}\right)=v
$$

where $v$ is reaction rate in a particle, $D_{e S}$ is effective diffusion coefficient of substrate, $c_{S}$ is substrate concentration in the particle, and $r$ is radial coordinate. In case of enzyme catalyzed reactions governed by Michaelis-Menten kinetics, the reaction rate can be calculated as

$$
v=\frac{V_{\max } c_{S}}{K_{M}+c_{S}}
$$

Kinetic parameters in Eq. (2) are maximum reaction rate, $V_{\max }$, and Michaelis constant, $K_{M}$. Eq. (1) can be solved applying the following boundary conditions

$$
\begin{gathered}
r=0:\left(\frac{\partial c_{S}}{\partial \mathrm{r}}\right)=0 \\
r=R: c_{S}=c_{S p}
\end{gathered}
$$

where $\mathrm{R}$ is particle radius and $c_{S p}$ is substrate concentration at particle surface. Solution of Eq. (1) enables to calculate the overall rate of catalytic reaction, $v_{o}$, and so-called effectiveness factor, $\eta$, indicating the measure of reaction limitation by particle mass transfer

$$
\eta=\frac{v_{o}}{v_{k}}
$$

where $v_{k}$ is reaction rate calculated directly from the kinetic equation (Eq. (2)) introducing substrate concentration at the particle surface which, in case of negligible external mass transfer limitations, is the same as that in the liquid medium surrounding the particle called bulk concentration. At substrate concentrations significantly lower than the $K_{M}$ value, the reaction kinetics is simplified to the first order and Eq. (1) can be solved analytically. Then, the effectiveness factor can also be calculated very simply from the following equation (Satterfield, 1969)

$$
\eta=\frac{3}{\phi}\left(\frac{1}{\tanh \phi}-\frac{1}{\phi}\right)
$$

Symbol $\phi$ in Eq. (5) denotes Thiele modulus defined, according to the symbols in Eqs. (1) and (2), as

$$
\phi=R \sqrt{\frac{V_{\max }}{K_{M} D_{e S}}}
$$

Eq. (5) is not applicable in the whole range of substrate concentrations and generally the effectiveness factor calculation requires solving Eq. (1) numerically. However, the task can be simplified using solutions published and generalized in terms of modified Thiele modulus (Aris, 1975):

$$
\phi_{m}=\frac{R}{3 \sqrt{2}} \sqrt{\frac{V_{\max }}{D_{e S} c_{S p}}}\left(\frac{1}{1+\beta,}\right)\left[1+\beta \ln \left(\frac{1}{1+\beta,}\right)\right]^{-1 / 2}
$$

where parameter $\beta$ is the ratio of the Michaelis constant and substrate concentration at the particle surface

$$
\beta=\frac{K_{M}}{c_{S p}}
$$

Using modified Thiele modulus, the effectiveness factor can be calculated with sufficient precision from the first order kinetic approximation (Aris, 1975)

$$
\eta=\frac{1}{\phi_{m}}\left(\frac{1}{\tanh 3 \phi_{m}}-\frac{1}{3 \phi_{m}}\right)
$$

From the practical point of view, determination of the effectiveness factor simplifies the estimation of the reaction rate in the system with immobilized enzyme from Eq. (4)

$$
v_{o}=\eta v_{k}
$$

because the value of $v_{k}$ can be directly calculated from the Michaelis-Menten equation introducing the bulk substrate concentration. Eq. (10) was used to evaluate experimental reaction rates with several simplifications as explained later.

Based on the experimental results described below, it is necessary to extend the kinetic model of the inhibitory effect of substances in the plantain extracts. Therefore, the above described mathematical model was combined with the modified Michaelis-Menten equation:

$$
v=\frac{V_{\max }\left(1-\frac{c_{I}}{K_{I}}\right) c_{S}}{K_{M}+c_{S}}
$$

It was assumed that the inhibitor concentration is proportional to the glucose concentration, since 
this was proportional to the total concentration of the extract in the medium. According to this assumption, Eq. (11) was modified to the form

$$
v=\frac{V_{\max }\left(1-\frac{k c_{c 0}}{K_{I}}\right) c_{S}}{K_{M}+c_{S}}
$$

where $k$ is the proportionality constant between initial glucose concentration, $c_{S 0}$, and inhibitor concentration. For the sake of simplification it was assumed that the inhibitory compounds are not consumed in particles with immobilized cells and their concentration is the same in the whole particle. In such case, the modified Thiele modulus could be calculated using the value of $\mathrm{V}_{\max , I}$ instead of $V_{\max }$

$$
V_{\max , I}=V_{\max }\left(1-\frac{k c_{c 0}}{K_{I}}\right)
$$

The inhibition model used assumes that the cell activity decreases proportionally with the increasing inhibitor concentration and it is zero at the concentration equal to the $K_{I}$ value.

\section{Material and Methods}

\section{Plantain extraction}

Extracts used in experiments were prepared by the following method: $200 \mathrm{~g}$ of fresh leaves of Plantago lanceolata grown in the eastern part of Slovakia were mixed with 11 of boiling water. The mixture was then cooled down to laboratory temperature and disintegrated using a Waring blender. At the end, solid parts were separated from the extract by filtration and centrifugation. In order to use the same experimental material throughout the experiment, the procedure was repeated until a sufficient amount of the extract was prepared; extracts were pooled and evaporated to $5 \%$ of the initial volume by vacuum evaporator at $35{ }^{\circ} \mathrm{C}$. The extract concentrate was divided into small portions and stored frozen at $-70{ }^{\circ} \mathrm{C}$ until use.

\section{Yeast pre-cultivation}

All kinds of yeast were used after their cultivation in an YG-medium containing $20 \mathrm{~g} \mathrm{dm}^{-3}$ of glucose and $10 \mathrm{~g} \mathrm{dm}^{-3}$ of yeast extract, with $\mathrm{pH}$ adjusted to 7 by $\mathrm{NaOH}$ solution before the sterilization. Cells were cultivated for $24 \mathrm{~h}$ in $500 \mathrm{ml}$ culture flasks filled with $100 \mathrm{ml}$ of medium at $30^{\circ} \mathrm{C}$ and $180 \mathrm{rpm}$ on an orbital shaker. The produced biomass was separated by centrifugation and used immediately in further experiments.

\section{Glucose removal by free cells}

Standard conditions of glucose removal by free cells were as follows: extract concentrate was re-diluted to the initial volume by $0.05 \mathrm{~mol} \mathrm{dm}^{-3}$ of an acetate buffer solution, $50 \mathrm{ml}$ of the re-diluted extract in a $500 \mathrm{ml}$ culture flask were mixed with yeast cells to the final cell concentration of $2 \mathrm{~g} \mathrm{dm}^{-3}$ (dry weight) and fermented at $30^{\circ} \mathrm{C}$ and $180 \mathrm{rpm}$ on an orbital shaker while the glucose content was analyzed by HPLC in regular intervals.

\section{HPLC glucose and aucubin analysis}

Glucose analysis was performed with the HPLC system Agilent 1260 RID equipped with a Watrex column $250 \times 8 \mathrm{~mm}$, Polymer IEX $8 \mu \mathrm{m}, \mathrm{H}^{+}$form. The analysis was run in the isocratic mode using a

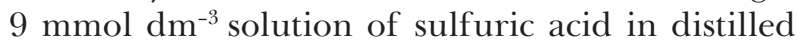
water. Flow rate of $0.7 \mathrm{ml} / \mathrm{min}$ and column temperature of $30{ }^{\circ} \mathrm{C}$ were applied. The quantification was performed by an RI-detector thermostated at $35^{\circ} \mathrm{C}$.

Aucubin analysis was done using the same HPLG system equipped with a Luna column C18, $250 \times 4.6 \mathrm{~mm} ; 5 \mu \mathrm{m}$ (Phenomenex). The analysis was run in gradient mode with mobile phases A: $0.1 \%$ of triflouoroacetic acid in $3 \%$ of methanol, and $\mathrm{B}$ : methanol : acetonitril (5:1). Gradient elution program used: $10 \mathrm{~min} 100 \% \mathrm{~A}$; linear gradient up to $95 \% \mathrm{~A}$ in $5 \mathrm{~min}$; up to $70 \% \mathrm{~A}$ in $15 \mathrm{~min}$; up to $70 \%$ $\mathrm{A}$ in $10 \mathrm{~min}$; up to $45 \% \mathrm{~A}$ in $12 \mathrm{~min}$; up to $100 \% \mathrm{~B}$ in $10 \mathrm{~min} ; 6 \mathrm{~min}$ isocratic, and equilibration with $100 \%$ A. Column temperature was $30^{\circ} \mathrm{C}$, injected volume was $1.5 \mu \mathrm{l}$, detection in $\mathrm{UV}$ was done at $210 \mathrm{~nm}$.

\section{Cell immobilization}

The volume of $50 \mathrm{ml}$ of $2 \%$ sodium alginate solution (Sigma-Aldrich) was stirred for complete alginate solubilization before adding the cells of Saccharomyces cerevisiae (DrOetker baker yeast) precultivated in the YG medium according to the above described procedure. Dry weight of biomass in the alginate solution was $5 \mathrm{~g} \mathrm{dm}^{-3}$. The mixture was homogenized by stirring and used for the preparation of particles by dropping the suspension through a syringe needle to a $2.5 \%$ solution of $\mathrm{CaCl}_{2}$. The particles were stirred in the solution for $20 \mathrm{~min}$ and used in further experiments.

\section{Glucose removal by immobilized cells}

The volume of $20 \mathrm{ml}$ of alginate particles with immobilized cells was packed in a jacketed glass column with the inner diameter of $1.6 \mathrm{~cm}$, between two $3 \mathrm{~cm}$ layers of glass beads insuring flow distribution. The packed bed reactor was used in the system with infinite medium recirculation depicted in Fig. 1 connected to a 11 fermenter (New Brunswick BioFlo/Celligen 115) equipped with peristaltic pumps for medium pumping and $\mathrm{pH}$ control, using a $1 \mathrm{~mol} \mathrm{dm}^{-3} \mathrm{NaOH}$ solution. The 


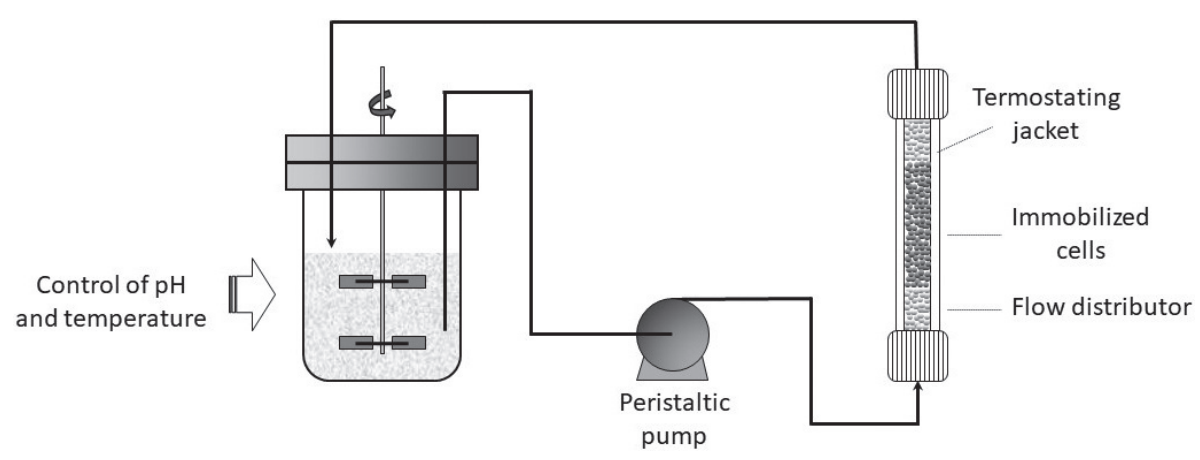

Fig. 1. Immobilized cell bioreactor system with infinite recirculation.

conditions in the fermenter system were: temperature of $30^{\circ} \mathrm{C}$, agitation of $400 \mathrm{rpm}, \mathrm{pH} 7$. Flow rate of medium through the packed bed reactor was $20 \mathrm{ml} \mathrm{min}^{-1}$. Before the measurement, the packed bed reactor was washed with a $0.05 \mathrm{~mol} \mathrm{dm}^{-3}$ acetate buffer, $\mathrm{pH} 7$, and the fermenter was filled with $500 \mathrm{ml}$ of the extract diluted 20 times with the same acetate buffer. The reaction was started by pumping the medium through the packed bed reactor. Samples of the medium were regularly taken from the stirred fermenter and analyzed by HPLC for glucose concentration.

\section{Results and Discussion}

Principal objective of this work was to find a suitable method for the removal of water-soluble saccharides from extracts of Plantago lanceolata, called plantain, to isolate bioactive compounds like aucubin. The procedure selected for this purpose was based on the ability of microbial cells, such as yeasts, to utilize various saccharides in the medium up to their complete consumption. Therefore, it is important to select a microorganism suitable for plantain extracts with respect to the content of compounds with potential effect on microbial cells.

\section{Microorganism screening}

Glucose removal in the extracts by free cells of various yeast strains was tested by fermentation in a set of shaking flasks inoculated by strains listed in Table 1. The obtained results show significant variability in the metabolic capacity of various strains, while the best capacity was observed with the tested Saccharomyces cerevisiae strain. Therefore, a strain of Saccharomyces cerevisiae was selected for the following experiments.

\section{Effect of pre-cultivation}

Preliminary experiments indicated that the ability of $S$. cerevisiae to metabolize glucose in the extracts varied during its cultivation. This was investigated
Tab. 1. Results of glucose removal in plantain extract by yeast strains within $24 \mathrm{~h}$ of fermentation.

\begin{tabular}{lc}
\hline Yeast strain & $\begin{array}{c}\text { Residual } \\
\text { glucose content } \\
(\%)\end{array}$ \\
\hline Rhodotorula aurantiaca CCY 20-9-1 & 32.9 \\
Debaryomyces hansenii NRRL Y-7483 & 35.8 \\
Metschnikowia reukaufii NRRL 6719 & 96.4 \\
Metschnikowia zobelii NRRL 5387 & 42.6 \\
Metschnikowia reukaufii NRRL 7112 & 57.9 \\
Saccharomyces oviformis RIVE 10-25-6 & 38.4 \\
Debaryomyces hansenii C-82129 & 30.7 \\
Pichia pastoris CGY 39-29-1 & 34.4 \\
Trigonopsis variabilis RIVE 24-1-1 & 28.4 \\
Kluyveromyces marxianus CCY 21-40-1 & 62.7 \\
Saccharomyces cerevisiae "DrOetker“ & 1.0 \\
\hline
\end{tabular}

by testing cells after different pre-cultivation times. From results summarized in Table 2 it is evident that the optimum pre-cultivation time was 24 hours.

Tab. 2. Extent of glucose removal in plantain extract by $S$. cerevisie as a function of the yeast pre-cultivation time.

\begin{tabular}{cc}
\hline $\begin{array}{c}\text { Pre-cultivation time } \\
(\mathbf{h})\end{array}$ & $\begin{array}{c}\text { Residual glucose content } \\
(\%)\end{array}$ \\
\hline 1 & 24.7 \\
8 & 13.9 \\
24 & 1.0 \\
\hline
\end{tabular}

There is one important observation that was made from this experiment: despite the well-known absolute ability of $S$. cerevisiae to completely utilize glucose, which is the best carbon and energy source for these cells, biomass pre-cultivated for 1 and 8 hours was not able to fully metabolize glucose in the plantain extract. 


\section{Extract toxicity toward cells}

The inability of cells to consume all glucose at certain conditions can be partially explained by the toxicity of certain compounds in plantain inhibiting their metabolic activities. The probability of this hypothesis was studied by a simple experiment set so that it was possible to compare the glucose consumption rate in media containing the same concentration of glucose but different amounts of plantain extract, i.e. different concentrations of supposedly inhibitory compounds. First, the concentrated plantain extract was diluted 20 times with a buffer solution to prepare a stock solution, and the glucose content in the solution was determined. Then, a series of extract dilutions was made by mixing the extract stock solution with different amounts of the buffer solution containing the same glucose concentration as in the stock extract solution. Media prepared in this way were inoculated with the S. cerevisiae biomass pre-cultured for $24 \mathrm{~h}$ and the glucose removal was tested at standard conditions described in Materials and Methods by a 1-hour long fermentation. Experimental results shown in Fig. 2 demonstrate that the extract content had a strong influence on the rate of glucose consumption.

The most significant difference was observed between the results corresponding to pure glucose solution and pure plantain extract (e.g. stock solution). Although also here, the glucose concentration was the same, the rate of glucose consumption in the pure extract was around 20 times lower than in the pure glucose solution.

\section{Immobilized cells system}

The use of free cells is not always convenient. When free cells of $S$. cerevisiae were reused after the glucose removal in the extract for another batch, their activity was comparable. Based on this it can be stated that the use of cells in their immobilized form can be advantageous. There are several reasons why the use of immobilized cells is preferred. Especially in the case under consideration, immobilized cells can easily be separated from the extract and they can be used repeatedly, which leads to savings in the cell preparation and isolation necessary before further purification steps. Therefore, the cells were immobilized in alginate particles and tested in a flowrecirculation reactor system for repeated batches of glucose removal from plantain extracts. Results of successive reaction courses are depicted in Fig. 3.

The catalytic system was very efficient in providing nearly total glucose removal in 4 hours. Repetition of batches within four days showed that instead of progressive cell degradation by inhibitory compounds in the extract, the reaction rate increased after each batch.

One important issue concerning the preparation of immobilized cell particles is the reasonable cell concentration in particles considering particle mass transfer limitations. This can be estimated based on the effectiveness factor. In regard to the relatively high dimensions of alginate particles with the average radius of $1.8 \mathrm{~mm}$, significant particle mass transfer limitations were expected. The reaction course was measured for a range of plantain extracts with different dilutions and the results

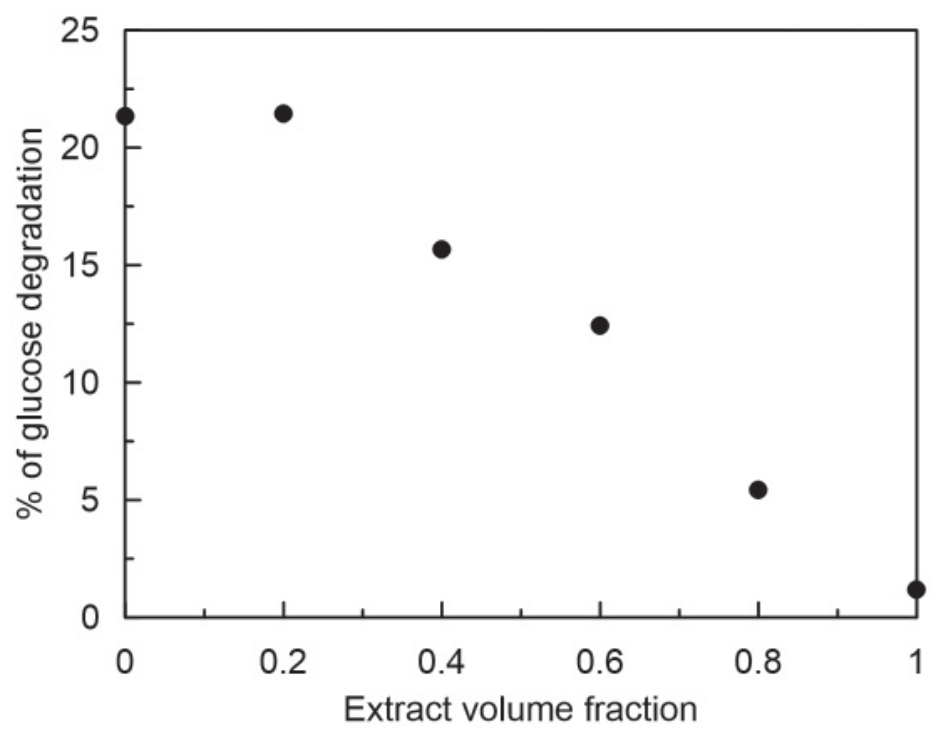

Fig. 2. Extent of glucose removal in media containing the same glucose concentration and variable plantain extract amounts. 


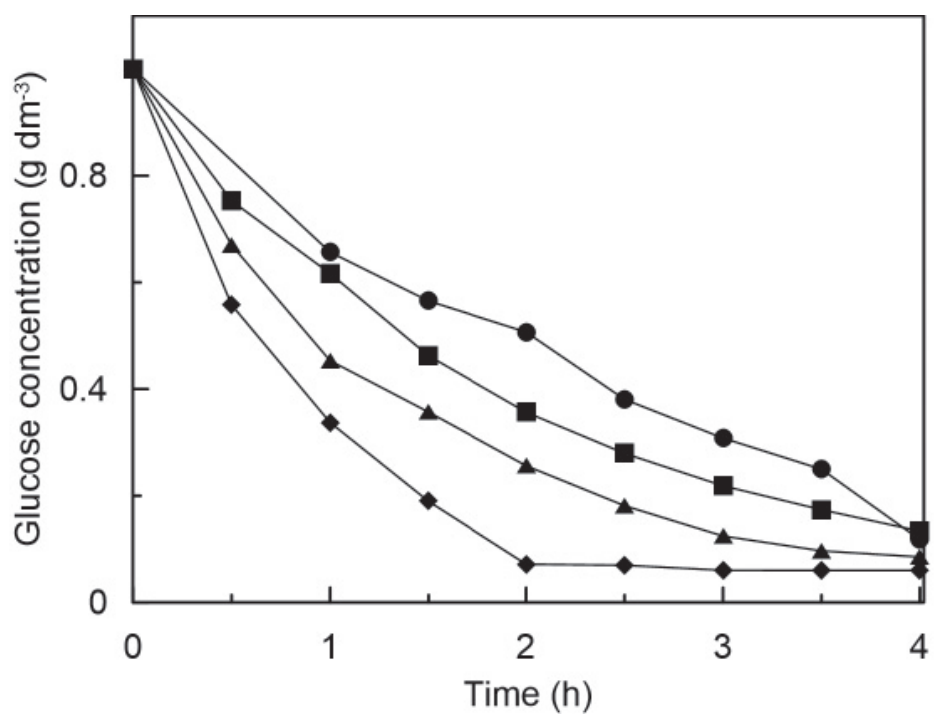

Fig. 3. Repeated batches of glucose degradation in the system with medium infinitive recirculation through the packed bed reactor system with alginate immobilized S. cerevisiae.

Order of batches: 1. - $\bullet$; 2. - $\mathbf{-}$; 3. - $\mathbf{\Delta}$; 4. -

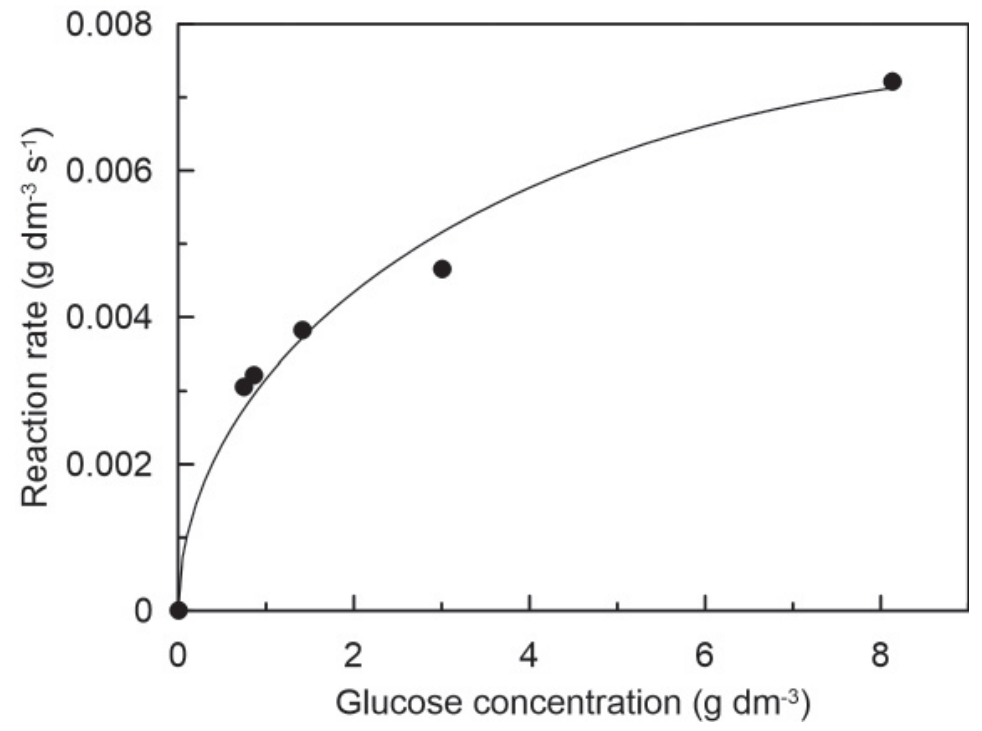

Fig. 4. Comparison of experimental and calculated initial rates of glucose degradation with immobilized cells of $S$. cerevisiae.

Corresponding parameter values were: $k / K_{I}=35.6 \mathrm{~g} / \mathrm{l}, V_{\max }=0.353 \mathrm{~g} / \mathrm{l} / \mathrm{s}, K_{M}=9 \cdot 10^{-9} \mathrm{~g} / \mathrm{l}$.

were used to calculate the initial reaction rates using a mathematical model. The whole reactor system with infinite recirculation was considered as a batch stirred reactor due to the very short resident time in the packed bed reactor (45 s) during which the change of the reaction rate was negligible, i.e. glucose concentration in the liquid phase was the same in the whole system, and depended only on time. Based on the experimental data, values of parameters in the model described above were calculated by nonlinear regression using the Microsoft Excel Solver. Glucose effective diffusion coefficient in alginate gel was $5.5 \cdot 10^{-12} \mathrm{~m}^{2} \mathrm{~s}^{-1}$ as taken from published data (Kashima and Imai, 2017).
Experimental and calculated reaction rates are shown in Fig. 4 with the resulting parameter values in figure caption. Introducing these values into the mathematic model, also the effectiveness factor was calculated as shown in Fig. 5.

Interestingly, the calculated value of $K_{M} 9 \cdot 10^{-9} \mathrm{~g} / 1$ was extremely low; significantly lower than the value presented in literature for glucose utilization by cells of Saccharomyces cerevisiae (Käppeli, 1987; van Urk et al., 1989; Weusthuis et al., 1994).

This can be explained by the fact that at given glucose concentrations, cells functioned at a nearly 0 -th order kinetics region and from the point of view of parameter calculation by nonlinear regres- 


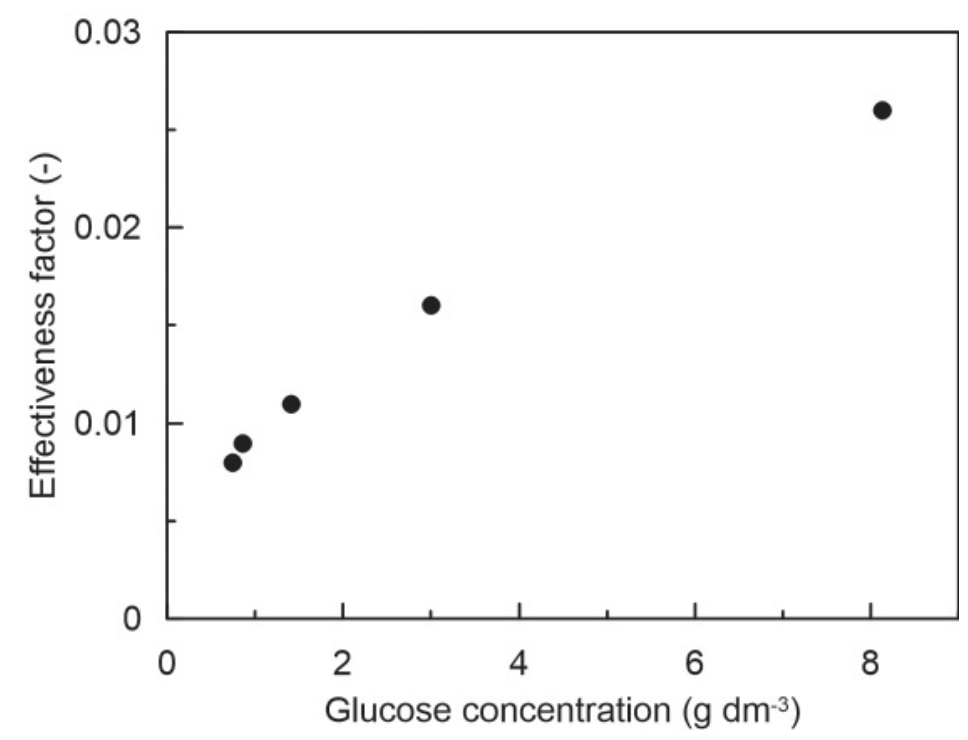

Fig. 5. Effectiveness factor as a function of initial glucose concentration.

sion from the experimental data, the optimization procedure simply tended towards the region of solution where $K_{M}$ has no meaning and can be omitted in the kinetic model.

Calculated values of the effectiveness factor shown in Fig. 5 were lower than 0.1. Considering that at negligible mass transfer limitations, the value of effectiveness factor is 1 , actual values were very low and only cells near the particle surface were involved in the reaction while the other ones were not active. Therefore it can be concluded that for optimum immobilized biocatalyst preparation, it is more economical to use lower cell concentrations to reduce the mass transfer limitations and enable more efficient use of all immobilized cells.

\section{Aucubin content in the extract}

An important criterion of applicability of the described approach for glucose degradation is the preservation of aucubin in the treated material. According to the analysis results, aucubin content after the treatment with $S$. cerevisiae was never reduced by more than $5 \%$ compared to the content in the original plantain extract and thus it can be concluded that the method described can significantly simplify aucubin purification.

\section{Acknowledgements}

This work was supported by the Slovak Research and Development Agency APVV-14-0538.

\section{References}

Aris R (1975) The mathematical theory of diffusion and reaction in permeable catalysts vol 1. Oxford University Press, New York.

Bermejo Benito P, Diaz Lanza AM, Silvan Sen AM, De Santos Galindez J, Fernandez Matellano L, Sanz Gomez A, Abad Martinez MJ (2000) Planta Med 66: 324-328.

Galvez M, Martin-Cordero C, Ayuso MJ (2005) J Enzyme Inhib Med Chem 20: 389-392.

Gonda S, Toth L, Parizsa P, Nyitrai M, Vasas G (2010) Acta Biol Hung 61 Suppl: 25-34.

Chang I-M (1997) Phytother Res 11: 189-192.

Kashima K, Imai M (2017) Food Bioprod Proces 102: 213-221.

Käppeli O (1987) Regulation of Carbon Metabolism in Saccharomyces cerevisiae and Related Yeasts. In: Rose AH, Tempest DW (eds) Advances in Microbial Physiology, vol 28. Academic Press, pp 181-209.

Kupeli E, Tatli II, Akdemir ZS, Yesilada E (2007) J Ethnopharmacol 110: 444-450.

Satterfield CN (1969) Mass transfer in heterogeneous catalysis. M.I.T. Press, Cambridge, Mass.

van Urk H, Postma E, Scheffers WA, van Dijken JP (1989) J Gen Microbiol 135: 2399-2406.

Weusthuis RA, Pronk JT, van den Broek PJ, van Dijken JP (1994) Microbiol Rev 58: 616-630. 